\title{
Relative Abundance and Species Composition of Different Predatory Ant Fauna at Sprayed and Unsprayed Areas in Brinjal Crop
}

\author{
S. Murali ${ }^{*}$, S.K. Jalali ${ }^{2}$, A.N. Shylesha ${ }^{2}$, T.M. Shivalinga Swamy ${ }^{2}$ and K.S. Jagadish ${ }^{3}$ \\ ${ }^{1}$ CSB, RSRS, Jammu - 181101, J \& K, India \\ ${ }^{2}$ NBAIR, Bengaluru - 560024, Karnataka, India \\ ${ }^{3}$ Agricultural Entomology, GKVK, Bengaluru - 560065, Karnataka, India \\ *Corresponding author
}

\begin{tabular}{|c|c|}
\hline & A B S T R A C T \\
\hline Keywords & \multirow{6}{*}{ 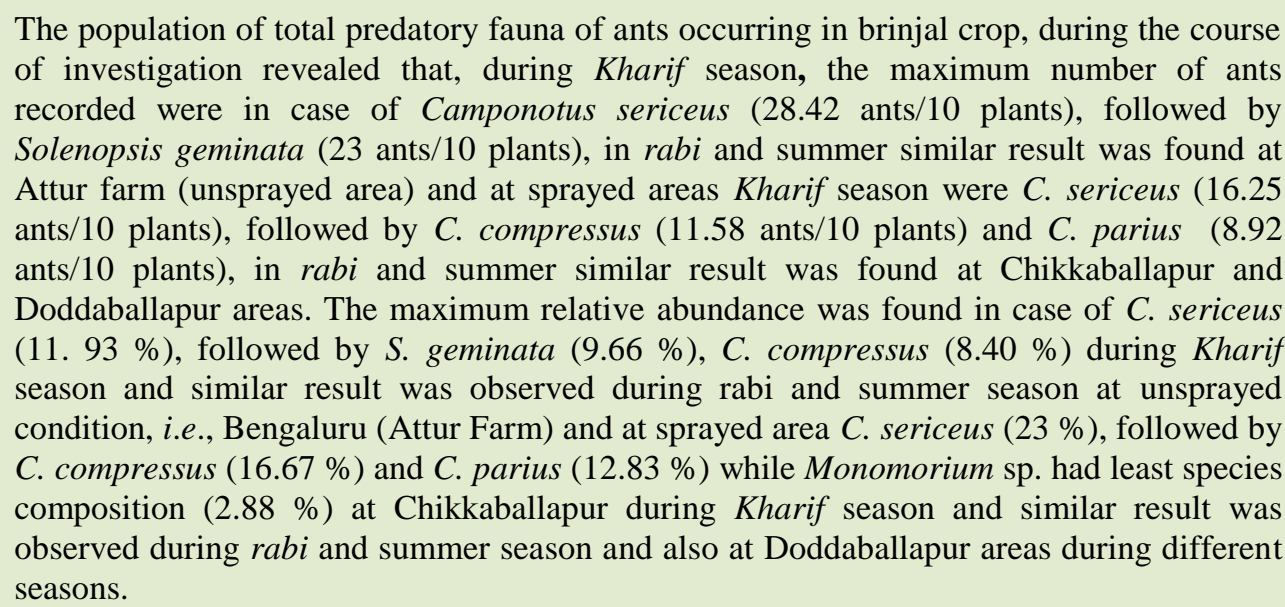 } \\
\hline $\begin{array}{l}\text { Brinjal, Sprayed } \\
\text { areas, Unsprayed } \\
\text { area, Ants, }\end{array}$ & \\
\hline $\begin{array}{l}\text { Seasons, Relative } \\
\text { abundance, }\end{array}$ & \\
\hline $\begin{array}{l}\text { Species } \\
\text { composition. }\end{array}$ & \\
\hline Article Info & \\
\hline $\begin{array}{l}\text { Accepted: } \\
23 \text { June } 2017 \\
\text { Available Online: } \\
\text { 10 July } 2017\end{array}$ & \\
\hline
\end{tabular}

\section{Introduction}

Arthropods are important components of ecosystems occupying vital positions in food webs, dynamics of populations and communities. They play various roles in ecosystems acting as herbivores, predators, decomposers, parasitoids and pollinators (Weaver, 1995). Added advantage is that they can be sampled quickly and reliably using various survey methods (New, 1998). Thus, arthropods are often used as biological indicators of ecosystem integrity (Tscharntke et al., 1998) and could be used reliably to infer ecosystem function and habitat condition (McGeoch, 1998; Weisser and Siemann, 2004). Population ecologists discussed diversity of arthropods in two aspects, species richness (i.e. the number of species in a set of samples) and equitability e.g., the number of individuals of each species in a sample (Disney, 1999). Brinjal, Solanum melongena L., is one of the three most important vegetables in many South Asian countries like India, Bangladesh, Nepal and Sri Lanka accounting for almost 50 per cent of the world's area under cultivation (Alam et al., 2003). In the brinjal field, various 
arthropod species both pests and natural enemies prevail from seedling to harvesting stage.

\section{Materials and Methods}

The documentation of natural enemy diversity was carried out in two systems, one unsprayed for which crop was raised at research farm of NBAIR, Bengaluru, at their Yelahanka Campus, Attur Farm and another at sprayed situation at farmers field at Doddaballapur and Chikkaballapur. The crop was raised/ observed from June, 2013 to June, 2014, for one year. At NBAIR research farm, plot was prepared by ploughing and crossploughing followed by laddering. All the plots were prepared with proper proportions of manure and fertilizers. The plot size was prepared $8 \times 8 \mathrm{~m}$ having $75 \times 60 \mathrm{~cm}$ plant spacing as control plot. The variety which we have used for experimentation was MAHYCO-11 throughout the year. For comparison from control plot, farmer fields were selected at Doddaballapur and Chikkaballapur districts. At farmers' field, the crop stage selected was 35 days after transplanting of the crop and plot size was $8 \times$ $8 \mathrm{~m}$ was selected irrespective of the plant spacing and variety grown by the farmers. The counting of predatory diversity, individuals were counted by using absolute methods like visual searching method by recording on plants as well as collection of various stages of predators. After collection, specimens were identified with the help of specialists. The sampling was done once in 10 days in both unsprayed and sprayed fields, thus 36 observations were recorded in a year.

\section{Results and Discussion}

During Kharif season at Bengaluru (Attur Farm) unsprayed area, the maximum number of ants recorded were in case of Camponotus sericeus (28.42 ants/10 plants), followed by
Solenopsis geminata (23 ants/10 plants), other major species recorded were $C$. compressus (20 ants/10 plants), Monomorium indicum (17.25 ants/10 plants), Myrmicaria brunnea (17.00 ants/10 plants), Monomorium sp. (16.92 ants/10 plants), M. criniceps (16.00 ants/10 plants), Crematogastor sp. (15.50 ants/ 10 plants), while in other species mean number ranged from 9.92 to 13.92 , whereas in minor species, mean number of ants recorded were 6.33 ants/10 plants (Table 1). Among the different ant species, maximum relative abundance was found in case of $C$. sericeus (11. $93 \%)$, followed by $S$. geminata $(9.66 \%)$, C. compressus $(8.40 \%)$, whereas in case of major species per cent composition ranged from 4.16 to 7.14 , however in minor species per cent composition was 2.66 of total diversity (Table 1).

Similarly, during rabi 2013-14, maximum number of ants recorded species-wise were $C$. sericeus (17.25 ants/10 plants), followed by $M$. indicum (11.67 ants/10 plants), $C$. compressus (11.25 ants/10 plants), $L$. processionalis (10.17 ants/10 plants), while in other species mean number ranged from 3.33 to 9.92 ants/10 plants in the decreasing order (Table 1). Among the ant species, maximum relative abundance was found in case of $C$. sericeus (12.03 \%), followed by $M$. indicum (8.14 \%), C. compressus (7.85 \%), L. processionalis $(7.09 \%), \quad P$. exercita, $L$. chinensis (6.92\%), M. criniceps $(6.57 \%), C$. parius $(6.51 \%), M$. bicolor $(6.16 \%)$ and Technomyrmex sp. which had least species composition $(2.33 \%)$ (Table 1). The maximum number of ants recorded specieswise during summer 2013-14 was for $C$. sericeus (22.42 ants/10 plants) followed by $C$. parius (17.67 ants/10 plants), C. compressus (14.58 ants/10 plants), M. brunnea (14.25 ants/10 plants), S. geminata (13.08 ants/10 plants), $M$. indicum (11.92 ants/10 plants), $M$. bicolor (11.33 ants/10 plants), $L$. processionalis (10.58 ants/10 plants), $P$. 
exercita (10.33 ants/10 plants), M. criniceps (10.25 ants/10 plants) and Technomyrmex sp. (5.92 ants/10 plants) in that decreasing order (Table 1). Among the ant species maximum relative abundance was recorded in case of $C$. sericeus (12.14\%) followed by $C$. parius (9.57 \%), C. compressus (7.90 \%), M. brunnea $(7.72 \%)$, followed by $S$. geminata (7.08 \%), M. indicum (6.45\%), M. bicolor $(6.14 \%)$, while Technomyrmex sp. was the least recorded species $(3.20 \%)$ (Table 1).

At Chikkaballapur sprayed area, maximum number of ant recorded species-wise in decreasing order during Kharif season were C. sericeus (16.25 ants/10 plants), followed by $C$. compressus (11.58 ants/10 plants) and C. parius (8.92 ants/10 plants) while Monomorium sp. was recorded in least numbers recorded (2.00 ants/10 plants) (Table 2). Among the ant species, maximum relative abundance was recorded in case of $C$. sericeus $(23 \%)$, followed by $C$. compressus $(16.67 \%)$ and $C$. parius $(12.83 \%)$ while Monomorium sp. had least species composition (2.88\%) (Table 2).

During rabi season, maximum number of ants recorded species-wise was in case of $C$. sericeus (10.17 ants/10 plants), followed by C. compressus (6.17 ants/10 plants), C. parius (6.08 ants/10 plants) and lowest number of ants recorded was in case of Technomyrmex sp. (1.25 ants/10plants) (Table 2). Among the ant species maximum species composition was found in case of $C$. sericeus (22\%), followed by $C$. compressus (13.43\%), $C$. parius $(13.25 \%)$ and the lowest species composition was observed in case of Technomyrmex sp. (2.72\%) (Table 2).

Maximum mean number of ant species was recorded during summer season, $C$. sericeus was recorded in significantly highest numbers (15.33 ants/10 plants), followed by $C$. compressus (9.25 ants/10 plants), C. parius (7.25 ants/10 plants), other ants (6.00 ants/10 plants), while Technomyrmex sp. had least mean number (2.50 ants/10 plants) (Table 2). Among the ant species maximum species composition was recorded in case of $C$. sericeus $(23.41 \%)$, followed by $C$. compressus (14.12\%), C. parius (11.07\%), other ants $(9.16 \%)$, while Technomyrmex sp. had lowest species composition (3.82 \%) (Table 2).

At Doddaballapur sprayed area, during Kharif season, the composition of ant species recorded was $C$. sericeus (13.08 ants/10 plants), followed by C. compressus (10.42 ants/10 plants), $C$. parius (7.17 ants/10 plants), M. brunnea (5.75 ants/10 plants), while Technomyrmex sp. had lowest composition of ants mean (1.83 ants/10 plants) (Table 3). Further relative abundance of ant species was as follows $C$. sericeus (21.33\%), followed by C. compressus (16.98 $\%)$, C. parius (11.68 \%), M. brunnea (9.38 $\%)$, whereas Technomyrmex sp. had least composition (2.99\%) (Table 3).

Species-wise mean number of ants recorded during rabi crop was as follows $C$. sericeus (11.00 ants/10 plants) followed by $C$. compressus (7.08 ants/10 plants), C. parius (5.42 ants/10 plants) and Monomorium sp. which showed lowest mean number of ants (2.42 ants/10 plants) (Table 3). Among the ant species maximum relative abundance was recorded in case of $C$. sericeus $(20.92 \%)$, followed by $C$. compressus (13.47\%), $C$. parius $(10.30 \%)$ and Monomorium sp. was recorded as having least abundance $(4.60 \%)$ (Table 3). During summer season, maximum mean number of ant species recorded were $C$. sericeus (14.42 ants/10 plants), followed by C. compressus (10.17 ants/10 plants), $M$. brunnea (7.25 ants/10 plants), C. parius (6.75 ants/10 plants), $C$. irritans (5.08 ants/10 plants) and lowest mean number of ants was found in case of Crematogaster sp. (2.58 ants/10 plants) (Table 3). 
Table.1 Relative abundance of different predatory ants in unsprayed area of brinjal crop at Attur Farm

\begin{tabular}{|c|c|c|c|c|c|c|c|c|c|}
\hline \multirow[b]{2}{*}{ Ant species } & \multicolumn{3}{|c|}{ Kharif } & \multicolumn{3}{|c|}{$R a b i$} & \multicolumn{3}{|c|}{ Summer } \\
\hline & Total & Mean \pm SD & $\begin{array}{c}\% \text { species } \\
\text { composition }\end{array}$ & Total & Mean \pm SD & $\begin{array}{c}\% \text { species } \\
\text { composition }\end{array}$ & Total & Mean \pm SD & $\begin{array}{c}\% \text { species } \\
\text { composition }\end{array}$ \\
\hline Camponotus compressus & 240.00 & $20.00 \pm 5.26$ & 8.40 & 135.00 & $11.25 \pm 2.53$ & 7.85 & 175.00 & $14.58 \pm 2.15$ & 7.90 \\
\hline Camponotus irritans & 146.00 & $12.17 \pm 3.69$ & 5.11 & 102.00 & $8.50 \pm 2.68$ & 5.93 & 118.00 & $9.83 \pm 4.61$ & 5.32 \\
\hline Camponotus parius & 167.00 & $13.92 \pm 2.68$ & 5.84 & 112.00 & $9.33 \pm 3.63$ & 6.51 & 212.00 & $17.67 \pm 6.08$ & 9.57 \\
\hline Camponotus sericeus & 341.00 & $28.42 \pm 10.26$ & 11.93 & 207.00 & $17.25 \pm 6.20$ & 12.03 & 269.00 & $22.42 \pm 5.28$ & 12.14 \\
\hline Crematogastor sp. & 186.00 & $15.50 \pm 5.45$ & 6.51 & 70.00 & $5.83 \pm 2.12$ & 4.07 & 82.00 & $6.83 \pm 1.95$ & 3.70 \\
\hline Leptogenys chinensis & 119.00 & $9.92 \pm 3.03$ & 4.16 & 119.00 & $9.92 \pm 3.03$ & 6.92 & 116.00 & $9.67 \pm 2.57$ & 5.23 \\
\hline Leptogenys processionalis & 122.00 & $10.17 \pm 3.35$ & 4.27 & 122.00 & $10.17 \pm 3.35$ & 7.09 & 127.00 & $10.58 \pm 2.87$ & 5.73 \\
\hline Meranoplus bicolor & 138.00 & $11.50 \pm 2.50$ & 4.83 & 106.00 & $8.83 \pm 2.52$ & 6.16 & 136.00 & $11.33 \pm 2.74$ & 6.14 \\
\hline Monomorium criniceps & 192.00 & $16.00 \pm 3.46$ & 6.72 & 113.00 & $9.42 \pm 2.50$ & 6.57 & 123.00 & $10.25 \pm 3.67$ & 5.55 \\
\hline Monomorium indicum & 207.00 & $17.25 \pm 6.20$ & 7.24 & 140.00 & $11.67 \pm 4.19$ & 8.14 & 143.00 & $11.92 \pm 3.90$ & 6.45 \\
\hline Monomorium sp. & 203.00 & $16.92 \pm 4.44$ & 7.10 & 76.00 & $6.33 \pm 1.56$ & 4.42 & 108.00 & $9.00 \pm 2.13$ & 4.87 \\
\hline Myrmicaria brunnea & 204.00 & $17.00 \pm 4.31$ & 7.14 & 87.00 & $7.25 \pm 1.48$ & 5.06 & 171.00 & $14.25 \pm 3.36$ & 7.72 \\
\hline Polyrhachis exercita & 119.00 & $9.92 \pm 2.94$ & 4.16 & 119.00 & $9.92 \pm 2.94$ & 6.92 & 124.00 & $10.33 \pm 2.46$ & 5.60 \\
\hline Solenopsis geminata & 276.00 & $23.00 \pm 4.99$ & 9.66 & 96.00 & $8.00 \pm 2.73$ & 5.58 & 157.00 & $13.08 \pm 3.63$ & 7.08 \\
\hline Technomyrmex sp. & 122.00 & $10.17 \pm 3.21$ & 4.27 & 40.00 & $3.33 \pm 2.06$ & 2.33 & 71.00 & $5.92 \pm 2.97$ & 3.20 \\
\hline Others & 76.00 & $6.33 \pm 1.56$ & 2.66 & 76.00 & $6.33 \pm 1.56$ & 4.42 & 84.00 & $7.00 \pm 1.21$ & 3.79 \\
\hline Total & 2858.00 & $238.17 \pm 36.79$ & 100.00 & 1720.00 & $143.33 \pm 20.39$ & 100.00 & 2216.00 & $184.67 \pm 24.18$ & 100.00 \\
\hline
\end{tabular}


Table.2 Relative abundance of different predatory ants in sprayed area of brinjal crop at Chikkaballapur

\begin{tabular}{|c|c|c|c|c|c|c|c|c|c|}
\hline \multirow[b]{2}{*}{ Ant species } & \multicolumn{3}{|c|}{ Kharif } & \multicolumn{3}{|c|}{$R a b i$} & \multicolumn{3}{|c|}{ Summer } \\
\hline & Total & Mean \pm SD & $\begin{array}{c}\% \text { species } \\
\text { composition }\end{array}$ & Total & Mean \pm SD & $\begin{array}{c}\% \text { species } \\
\text { composition }\end{array}$ & Total & Mean \pm SD & $\begin{array}{c}\% \text { species } \\
\text { composition }\end{array}$ \\
\hline Camponotus compressus & 139.00 & $11.58 \pm 4.38$ & 16.67 & 74.00 & $6.17 \pm 1.27$ & 13.43 & 111 & $9.25 \pm 3.22$ & 14.12 \\
\hline Camponotus irritans & 38.00 & $3.17 \pm 0.94$ & 4.56 & 35.00 & $2.92 \pm 0.79$ & 6.35 & 48 & $4.00 \pm 0.95$ & 6.11 \\
\hline Camponotus parius & 107.00 & $8.92 \pm 3.00$ & 12.83 & 73.00 & $6.08 \pm 1.78$ & 13.25 & 87 & $7.25 \pm 2.80$ & 11.07 \\
\hline Camponotus sericeus & 195.00 & $16.25 \pm 4.25$ & 23.38 & 122.00 & $10.17 \pm 2.55$ & 22.14 & 184 & $15.33 \pm 3.52$ & 23.41 \\
\hline Crematogastor sp. & 40.00 & $3.33 \pm 1.07$ & 4.80 & 26.00 & $2.17 \pm 0.58$ & 4.72 & 54 & $4.50 \pm 1.51$ & 6.87 \\
\hline Meranoplus bicolor & 50.00 & $4.17 \pm 1.19$ & 6.00 & 22.00 & $1.83 \pm 0.83$ & 3.99 & 36 & $3.00 \pm 1.48$ & 4.58 \\
\hline Monomorium criniceps & 48.00 & $4.00 \pm 1.71$ & 5.76 & 38.00 & $3.17 \pm 0.83$ & 6.90 & 39 & $3.25 \pm 0.87$ & 4.96 \\
\hline Monomorium sp. & 24.00 & $2.00 \pm 0.85$ & 2.88 & 22.00 & $1.83 \pm 0.83$ & 3.99 & 32 & $2.67 \pm 1.44$ & 4.07 \\
\hline Myrmicaria brunnea & 62.00 & $5.17 \pm 1.19$ & 7.43 & 40.00 & $3.33 \pm 0.65$ & 7.26 & 41 & $3.42 \pm 0.67$ & 5.22 \\
\hline Solenopsis geminata & 52.00 & $4.33 \pm 1.23$ & 6.24 & 42.00 & $3.50 \pm 0.52$ & 7.62 & 52 & $4.33 \pm 1.37$ & 6.62 \\
\hline Technomyrmex sp. & 38.00 & $3.17 \pm 1.59$ & 4.56 & 15.00 & $1.25 \pm 0.45$ & 2.72 & 30 & $2.50 \pm 0.80$ & 3.82 \\
\hline Others & 41.00 & $3.42 \pm 1.08$ & 4.92 & 42.00 & $3.50 \pm 1.09$ & 7.62 & 72 & $6.00 \pm 1.28$ & 9.16 \\
\hline Total & 834.00 & $69.50 \pm 11.04$ & 100.00 & 551.00 & $45.92 \pm 4.52$ & 100.00 & 786 & $65.50 \pm 8.46$ & 100 \\
\hline
\end{tabular}


Table.3 Relative abundance of different predatory ants in sprayed area of brinjal crop at Doddaballapur

\begin{tabular}{|c|c|c|c|c|c|c|c|c|c|}
\hline \multirow[b]{2}{*}{ Ant species } & \multicolumn{3}{|c|}{ Kharif } & \multicolumn{3}{|c|}{ Rabi } & \multicolumn{3}{|c|}{ Summer } \\
\hline & Total & Mean \pm SD & $\begin{array}{c}\% \text { species } \\
\text { composition }\end{array}$ & Total & Mean \pm SD & $\begin{array}{c}\% \text { species } \\
\text { composition }\end{array}$ & Total & Mean \pm SD & $\begin{array}{c}\% \text { species } \\
\text { composition }\end{array}$ \\
\hline Camponotus compressus & 125.00 & $10.42 \pm 3.40$ & 16.98 & 85.00 & $7.08 \pm 1.16$ & 13.47 & 122.00 & $10.17 \pm 1.90$ & 14.63 \\
\hline Camponotus irritans & 49.00 & $4.08 \pm 1.51$ & 6.66 & 44.00 & $3.67 \pm 1.30$ & 6.97 & 61.00 & $5.08 \pm 2.02$ & 7.31 \\
\hline Camponotus parius & 86.00 & $7.17 \pm 3.16$ & 11.68 & 65.00 & $5.42 \pm 1.44$ & 10.30 & 81.00 & $6.75 \pm 2.86$ & 9.71 \\
\hline Camponotus sericeus & 157.00 & $13.08 \pm 1.88$ & 21.33 & 132.00 & $11.00 \pm 2.49$ & 20.92 & 173.00 & $14.42 \pm 3.42$ & 20.74 \\
\hline Crematogastor sp. & 28.00 & $2.33 \pm 0.49$ & 3.80 & 32.00 & $2.67 \pm 1.23$ & 5.07 & 31.00 & $2.58 \pm 0.90$ & 3.72 \\
\hline Meranoplus bicolor & 36.00 & $3.00 \pm 0.74$ & 4.89 & 33.00 & $2.75 \pm 1.14$ & 5.23 & 40.00 & $3.33 \pm 1.50$ & 4.80 \\
\hline Monomorium criniceps & 42.00 & $3.50 \pm 1.24$ & 5.71 & 42.00 & $3.50 \pm 0.90$ & 6.66 & 46.00 & $3.83 \pm 1.19$ & 5.52 \\
\hline Monomorium sp. & 24.00 & $2.00 \pm 0.85$ & 3.26 & 29.00 & $2.42 \pm 1.08$ & 4.60 & 43.00 & $3.58 \pm 1.16$ & 5.16 \\
\hline Myrmicaria brunnea & 69.00 & $5.75 \pm 1.76$ & 9.38 & 54.00 & $4.50 \pm 1.62$ & 8.56 & 87.00 & $7.25 \pm 2.01$ & 10.43 \\
\hline Solenopsis geminata & 47.00 & $3.92 \pm 1.00$ & 6.39 & 48.00 & $4.00 \pm 0.74$ & 7.61 & 53.00 & $4.42 \pm 1.44$ & 6.35 \\
\hline Technomyrmex sp. & 22.00 & $1.83 \pm 0.72$ & 2.99 & 31.00 & $2.58 \pm 1.16$ & 4.91 & 44.00 & $3.67 \pm 0.78$ & 5.28 \\
\hline Others & 51.00 & $4.25 \pm 1.76$ & 6.93 & 36.00 & $3.00 \pm 1.21$ & 5.71 & 53.00 & $4.42 \pm 1.16$ & 6.35 \\
\hline Total & 736.00 & $61.33 \pm 8.53$ & 100.00 & 631.00 & $52.58 \pm 5.11$ & 100.00 & 834.00 & $69.50 \pm 9.65$ & 100.00 \\
\hline
\end{tabular}


Among the ant species, maximum relative abundance was found in case of $C$. sericeus (20.74\%), followed by C. compressus (14.63 $\%)$, M. brunnea (10.43 \%), C. parius (9.71 $\%), \quad C$. irritans $(7.31 \%)$ and lowest abundance recorded was in case of Crematogaster sp. (3.72\%) (Table 3).

The results of the plant dwelling predaceous and other insects in the present study suggest that ants, spiders and coleopterans are the most important predators on brinjal crop. The present findings closely agree with the findings of El-Shafie (2001), who observed 28 species of insect pests under seven different insect orders and coleopteran and ants as major predaceous insects, while Nayar et al., (1995) reported 53 species of insect pests of brinjal. Assessment of parasitoid community is one of the key steps of understanding pest dynamics. Successful biological control program requires detailed studies on biology and behaviour of the host and their natural enemies, interaction among hosts, natural enemies and environment. Although, several researchers published reports on pest of brinjal elsewhere however, information about total arthropods community in the brinjal agro-ecosystem is limited. So, our objective was to observe the arthropod biodiversity in the brinjal agro-ecosystem both under unsprayed and sprayed conditions.

The population of the total predatory fauna occurring in brinjal crop, viz., ants, spiders, coccinellids, green lacewing, assassin bugs, wasps, praying mantis, earwigs and others were recorded during the course of this investigation. Twenty-one species of ants were found to occur in the brinjal crop, in which $M$. brunnea, $S$. geminata and $M$. criniceps were recorded as the dominant species both under unsprayed and sprayed conditions. Present investigation is in agreement with earlier report of Latif et al., (2009), who recorded 17 species of ants, among them 7 families were identified as predators, while El-Shafie (2001) observed 28 species of insect pests under seven different insect orders from the brinjal ecosystem and Nayar et al., (1995) reported 53 species of insect pests of brinjal.

\section{Acknowledgements}

The authors wish to thank the authorities of National Bureau of Agricultural Insect Resources (NBAIR), Bengaluru - 560024, Karnataka, India for their facilities to undertake this work. We acknowledge all technical staff who assisted their help in successful completion of work and also farmers who have cooperated for collection of field samples at different places.

\section{References}

Alam, S.N., Rashid, M.A., Rouf, F.M.A., Jhala, R.C., Patel, J.R., Satpathy, S., Shivalingaswamy, T.M., Rai, S, Wahundeniya, I., Cork, A., Ammaranan, C. and Talekar, N. S. 2003. Development of an integrated pest management strategy for eggplant fruit and shoot borer in South Asia. Technical Bulletin, AVRDC. The World Vegetable Center, Shanhua, Taiwan, 28: 1-66.

Disney, R.H.L. 1999. Insect biodiversity and demise of alpha taxonomy. Antenna: Bull. R. Entomol. Soc., 23: 84-88.

El-Shafie, H.A.F. 2001. The use of neem products for sustainable management of homopterous key pests on potato and eggplant in the Sudan. Ph. D. Thesis, Institute of Phytopathology and Applied Zoology Experimental station Justus Liebig University of Giessen, Germany.

Latif, M.A., Rahman, M.M., Alam, M.Z. and Hossain, M.M. 2009. Evaluation of flubendiamide as an IPM component for the management of brinjal shoot and 
fruit borer, Leucinodes orbonalis Guenee. Munis Entomol. Zool., 4(1): 257-267.

Mcgeoch, M.A. 1998. The selection, testing and application of terrestrial insects as bioindicators. Biol. Rev., 73: 181-201.

Nayar, K.K., Ananthakrishnan, T.N. and David, B.V. 1995. General and Appl. Entomol., 11th Edn. Tata McGraw-Hill Publ. Co. Ltd., New Delhi, India.

New, T.R. 1998. Invertebrate Surveys for Conservation. 1st Edn. Oxford University Press, New York.
Tscharntke, T.A., Gathmann and Dewenter, S.I. 1998. Bioindication using trapnesting bees and wasps and their natural enemies: Community structure and interactions. J. Appl. Ecol., 5: 708-719.

Weaver, J.C. 1995. Indicator species and scale of observation. Conser. Biol., 9: 939-942.

Weisser, W.W. and Sieman, E. 2004. Insects and ecosystem function. 1st Edn. Springer-erlag Berlin Heidelberg, New York.

\section{How to cite this article:}

Murali, S., S.K. Jalali, A.N. Shylesha, T.M. Shivalinga Swamy and Jagadish, K.S. 2017. Relative Abundance and Species Composition of Different Predatory Ant Fauna at Sprayed and Unsprayed Areas in Brinjal Crop. Int.J.Curr.Microbiol.App.Sci. 6(7): 2616-2623. doi: https://doi.org/10.20546/ijcmas.2017.607.309 